\title{
¿Estudias o trabajas? - El aprendizaje basado en proyectos como una experiencia enriquecedora de trabajar y estudiar.
}

\author{
L. Quiles-Carrillo ${ }^{a}$, V. Fombuena ${ }^{a}$, R. Balart ${ }^{\mathrm{a}}$, T. Boronat ${ }^{\mathrm{a}}$ y N. Montanes $^{\mathrm{a}}$ \\ ${ }^{a}$ Grupo de Innovación de Prácticas Académicas (GIPA), Universitat Politècnica de València, Plaza \\ Ferrándiz y Carbonell s/n, Alcoy, Alicante (ESPAÑA).

\begin{abstract}
Project Based Learning (PBL) is a very popular teaching strategy today since it offers a multitude of advantages and/or opportunities from a teaching point of view, such as achieving deeper learning, developing teamwork and leadership skills, or getting the student to take control of what should be learned and how it should be learned. For all these reasons the authors believe that in the context of Master's studies, as the last studies before insertion into working life, the PBL can be a very appropriate teaching methodology, as well thought out will prepare students for the real situations they will later live in the exercise of their professional life. The aim of this work is to expose the experience of the application of the PBL in a subject of the Master's Degree in Engineering, Processing and Characterization of Materials, taught at the Higher Polytechnic School of Alcoy, belonging to the Universitat Politècnica de València (UPV).
\end{abstract}

Keywords: Project-based learning (PBL), teamwork, active learning.

\section{Resumen}

El Aprendizaje Basado en Proyectos (ABP), también conocido como Project Based Learning (PBL), es una estrategia didáctica muy en auge en la actualidad debido a que ofrece una gran multitud de ventajas $y / u$ oportunidades desde el punto de vista de la docencia, como por ejemplo logar un aprendizaje más profundo, desarrollar el trabajo en equipo y las habilidades de liderazgo, o conseguir que el estudiante asuma el control de lo que se debe aprender y cómo se debe aprender. Por todos estos motivos los autores pensamos que, en el contexto de los estudios de Máster, como últimos estudios antes de la inserción en la vida laboral, el PBL puede ser una metodología docente muy adecuada, ya que bien planteada preparará a los alumnos ante las situaciones reales que luego vivirán en el ejercicio de su vida profesional. El objetivo de este trabajo es exponer la experiencia de la aplicación del PBL en una asignatura del Máster Universitario en Ingeniería, Procesado y Caracterización de Materiales, impartido en la Escuela Politécnica Superior de Alcoy, perteneciente a la Universitat Politècnica de València (UPV).

Palabras clave: Aprendizaje Basado en Proyectos (ABP), trabajo en equipo, aprendizaje activo. 
¿Estudias o trabajas? - El aprendizaje basado en proyectos como una experiencia enriquecedora de trabajar y estudiar.

\section{Introducción}

El Aprendizaje Basado en Proyectos (ABP), también conocido como Project Based Learning (PBL), es una estrategia didáctica muy en auge en la actualidad en la que los alumnos, combinados en grupos, llevan a término proyectos que se basan en situaciones reales.

En el marco del Espacio Europeo de Enseñanza Superior (EEES) el aprendizaje se centra en el estudiante. Los contenidos pasan a un segundo orden de prioridad, siendo que lo que realmente cobra importancia es la necesidad de enseñar a aprender y los métodos que se emplean para ello. El objetivo es preparar a las nuevas generaciones para el aprendizaje continuo (Reverte Bernabeu, Gallego et al., 2007). En este nuevo paradigma, el PBL nos ofrece una serie de ventajas $y / u$ oportunidades entre las que caben destacar:

- Produce un aprendizaje más profundo en los estudiantes.

- Logra que los alumnos se conviertan en los principales protagonistas de su propio proceso de aprendizaje.

- Promueve el trabajo en equipo y el liderazgo.

- Cambia el enfoque docente centrándolo en el desarrollo de las competencias y no en la memorización de conocimientos, lo que redundará en una mejor preparación de los estudiantes de cara a la vida laboral.

- Genera un ambiente docente activo, dinámico, en el que profesores y alumnos interactúan y hay camino para la retroalimentación, lo que al final genera una mejora del aprendizaje.

Por todas estas razones el PBL ha despertado mucho interés en la enseñanza, no solo en el ámbito de la educación superior, sino a distintos niveles educativos (Boud, 1985, SavinBaden, 2000, Kolmos, Kuru et al., 2007, Cascales Martínez, Carrillo García et al., 2017).

De Graaff y Ravesteijn postulan que el PBL permite desarrollar el trabajo en equipo, la resolución de problemas y las habilidades de liderazgo, dentro de un marco en el que el estudiante asume el control de lo que se debe aprender y cómo se debe aprender (De Graaff and Ravesteijn, 2001).

Por otra parte Schön sugiere que, en el ambiente laboral, los ingenieros reflexionan en acción, por lo que las habilidades necesarias a adquirir para hacer esto no se pueden enseñar en el aula o en el laboratorio, empleando solo teoría científica, sino efectuando un estudio de diseño (Schön, 1987, Schön, 2017).

Por todos estos motivos los autores pensamos que, en el contexto de los estudios de Máster, como últimos estudios antes de la inserción en la vida laboral, siendo que son pocos los alumnos que deciden tratar de doctorarse, el Aprendizaje Basado en Proyectos puede ser una metodología docente muy adecuada, ya que bien planteada, entre otras muchas cosas, preparará a los alumnos ante las situaciones reales que luego vivirán en el ejercicio de su vida profesional.

Así pues, el objetivo de este trabajo es exponer la experiencia de la aplicación del PBL en una asignatura del Máster Universitario en Ingeniería, Procesado y Caracterización de Materiales, impartido en la Escuela Politécnica Superior de Alcoy, perteneciente a la 
Universitat Politècnica de València (UPV), en concreto en la asignatura de "Estructura y técnicas de caracterización de materiales avanzados".

\section{Objetivos}

La asignatura referida, "Estructura y técnicas de caracterización de materiales avanzados", es una asignatura obligatoria del primer curso, semestre A, del Máster Universitario en Ingeniería, Procesado y Caracterización de Materiales. Tiene una carga total de 6 créditos, repartidos en 3 créditos de teoría y 3 créditos de prácticas. Presenta una media de 16 alumnos matriculados tomando como referencia los últimos 6 cursos académicos. El rendimiento académico desde el curso 2013/2014, hasta la actualidad, se sitúa en torno a un $90 \%$.

La asignatura está estructurada en dos bloques cuyos contenidos genéricos son los siguientes:

Bloque I: ESTRUCTURA Y APLICACIONES DE MATERIALES AVANZADOS. Materiales con comportamiento inteligente, materiales biomiméticos y materiales para industrias altamente tecnológicas.

Bloque II: TÉCNICAS DE CARACTERIZACIÓN DE MATERIALES AVANZADOS. Técnicas espectroscópicas, técnicas de análisis térmico, técnicas de caracterización mecánica y otras caracterizaciones. Fundamentos y aplicaciones de técnicas de caracterización para materiales avanzados.

\subsection{Objetivo general}

El objetivo general plantado por los autores es que los alumnos, combinados en grupos, desarrollen nuevos productos realizados con materiales avanzados. Para ello será necesario caracterizar completamente dichos materiales. La idea es abordar y trabajar los contenidos de los bloques referidos anteriormente mientras se desarrollan las distintas fases de los proyectos propuestos.

\subsection{Objetivos específicos}

Por otra parte, los objetivos específicos propuestos a los alumnos serán:

- Plantear un posible nuevo producto que precise estar elaborado con materiales avanzados.

- Formular un nuevo producto con materiales avanzados.

- Desarrollar un nuevo producto con materiales avanzados.

- Caracterizar los materiales avanzados empleados en el nuevo producto planteado.

Y en el marco de las Competencias Transversales de la UPV, los profesores pretenden:

- Desarrollar la innovación, creatividad y emprendimiento (CT04).

- Desarrollar el trabajo en equipo y liderazgo (CT06).

- Desarrollar la responsabilidad ética, medioambiental y profesional (CT07).

\subsection{Objetivos a largo plazo}

Cabe comentar por último que en el Máster referido se encuentran diversas asignaturas (en concreto 4) que giran en torno a los materiales avanzados, las técnicas no convencionales de 
¿Estudias o trabajas? - El aprendizaje basado en proyectos como una experiencia enriquecedora de trabajar y estudiar.

procesado de materiales, al diagnóstico y comportamiento en servicio de materiales y a las metodologías de investigación y desarrollo de nuevos proyectos. El objetivo final es lograr coordinar un proyecto que involucre a las 4 asignaturas.

\section{Desarrollo de la innovación}

La idea pues es vertebrar el aprendizaje a través de un proyecto que englobe todos los aspectos y conocimientos a tratar en la asignatura del Máster referida anteriormente, "Estructura y técnicas de caracterización de materiales avanzados".

Para ello los autores en un principio se propusieron modificar el curricula formativo de esta asignatura en las dimensiones de diseño curricular, desarrollo curricular y evaluación curricular, con el objetivo de lograr un alineamiento curricular, y para este fin se plantearon las siguientes preguntas: ¿Qué enseñar? ¿Cómo enseñar? ¿Cuándo enseñar? ¿Qué, cómo y cuándo evaluar?

La respuesta a ¿Qué enseñar? se puede responder a partir de la siguiente pregunta motriz planteada a nuestros alumnos: "¿Soy capaz de desarrollar y caracterizar completamente un nuevo producto con materiales avanzados?", así como de los objetivos específicos propuestos a los alumnos enumerados en el apartado anterior.

En cuanto a el ¿Cómo enseñar?, el PBL es la estrategia metodológica y didáctica que determina las actividades y los recursos necesarios, pero de manera general, también han sido empleadas los siguientes tipos de actividades y herramientas docentes:

- Puzzles: para que los estudiantes aprendan los temas teóricos. Se reparten dentro de cada grupo de alumnos los temas a estudiar. Después cada miembro del grupo toma el rol de profesor, explicando al resto de los compañeros del grupo el tema que le ha tocado estudiarse y asegurándose de que todos los miembros del grupo aprendan bien el tema en cuestión, que primeramente estudio él. Al final todos los miembros del grupo han ejercido roles de profesores y de alumnos, y se habrán aprendido todos los temas. Por otro lado, para el estudio de los temas los alumnos cuentan con un amplio repertorio de material docente preparado por los profesores responsables de la asignatura, incluidos los objetos de aprendizaje de Docencia en Red (Polimedias, Artículos Docentes, Screencast, etc.).

- Clases Expositivas: se hace necesario impartir un número limitado de ellas para solventar dudas que van surgiendo durante la realización del proyecto. Son de corta duración y únicamente orientadas a resolver los problemas que surgen durante el desarrollo del proyecto, en la fase que proceda.

La pregunta “¿Cuándo enseñar?” precisa del establecimiento por parte de los profesores responsables de la asignatura de un Plan de Trabajo semanal, detallado y minucioso, que contemple las actividades a realizar con referencia a los distintos grupos, las fechas para completar las diversas etapas, etc.

Y por último, en cuanto a “¿Qué, cómo y cuándo evaluar?”, las herramientas evaluativas usadas han sido las siguientes: 
- Autoevaluaciones. Son ejercicios individuales sobre los temas del proyecto que el alumno debe autoevaluar. Para ello dispone de una lista de condiciones a comprobar como, por ejemplo: ¿Qué pasaría si...?, ¿Qué diferencia hay entre ... y ...?, etc.

- Evaluación entre compañeros. Cada grupo de estudiantes evaluará 2 de los nuevos materiales avanzados resultantes de los productos presentados por los otros grupos (materiales a procesar y caracterizar en las etapas avanzadas del proyecto, los cuales se corresponden con los bloques propuestos - ver Fig. 1). Para la evaluación entre iguales los alumnos cuentan con una rubrica elaborada por los profesores responsables de la asignatura.

- Exámenes de comprobación de la adquisición de conocimientos mediante el empleo de la herramienta Kahoot.

- Entregables parciales.

- Entregable y defensa pública final.

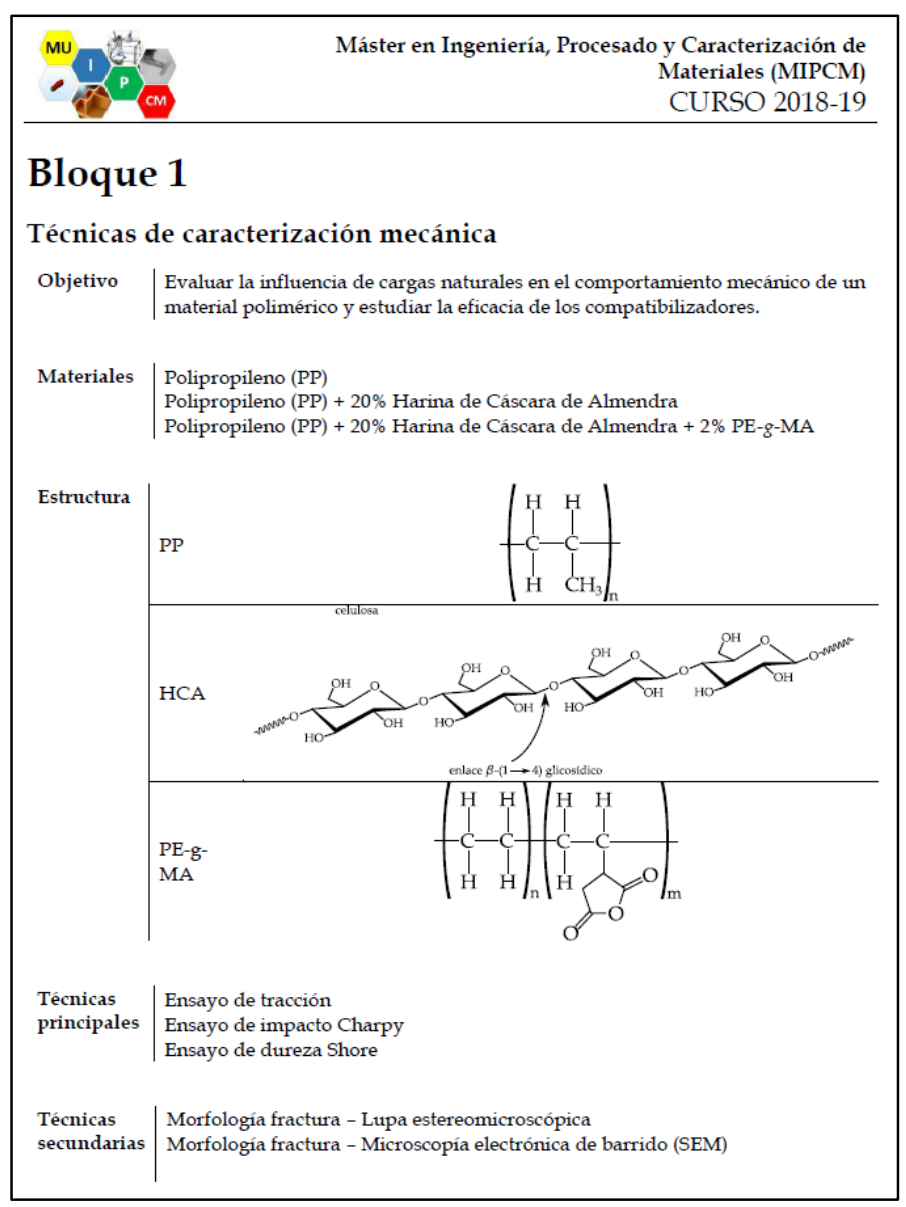

Fig. 1 Material avanzado Bloque 1.

Una vez se tuvo claro esto, el siguiente paso fue realizar una planificación detallada que englobara todos los aspectos y de la cual se desprendieran cada uno de los pasos a dar, que abordara con sumo detalle el desarrollo metodológico de la innovación. En la Tabla 1 se 
¿Estudias o trabajas? - El aprendizaje basado en proyectos como una experiencia enriquecedora de trabajar y estudiar.

muestra de manera resumida esa planificación elaborada para la implantación del PBL en la asignatura referida.

Tabla 1. Planificación para la implantación del PBL en la asignatura "Estructura y técnicas de caracterización de materiales avanzados"

\begin{tabular}{|c|c|c|}
\hline Semana & Presencial, en clase & No presencial, en casa \\
\hline 1 & $\begin{array}{l}\text { - } \\
\text { - } \quad \text { Instrucentación del proyecto. } \\
\text { - } \quad \text { Formación de los grupos. } \\
\text { - Presentación de los materiales docentes del } \\
\text { puzzle. }\end{array}$ & $\begin{array}{l}\text { - Reunión inicial grupos para intercambio de } \\
\text { impresiones. }\end{array}$ \\
\hline 2,3 y 4 & $\begin{array}{l}\text { - Clases expositivas para la resolución de } \\
\text { dudas concretas. } \\
\text { - Actividades de autoevaluación. }\end{array}$ & 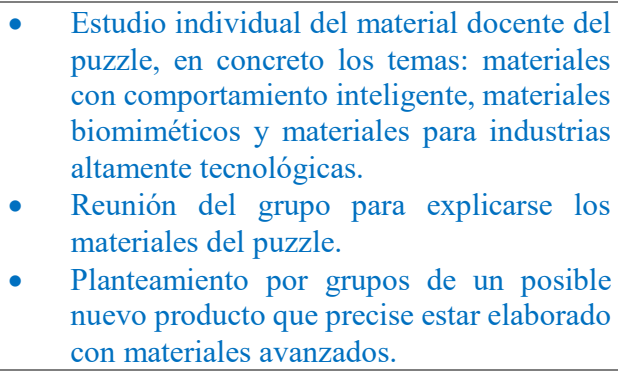 \\
\hline 5 & $\begin{array}{l}\text { - Repaso requerimientos y solicitaciones que } \\
\text { deben cumplir los posibles productos } \\
\text { planteados por los grupos. }\end{array}$ & $\begin{array}{l}\text { - Formulación y desarrollo de un nuevo } \\
\text { producto elaborado con materiales } \\
\text { avanzados. } \\
\text { Definición del nuevo material o de una } \\
\text { familia de materiales avanzados (cada } \\
\text { familia de nuevos materiales quedará } \\
\text { identificada y englobada en un "bloque de } \\
\text { trabajo"). Entregable 1. }\end{array}$ \\
\hline 6 & $\begin{array}{l}\text { Evaluación entre compañeros de los nuevos } \\
\text { materiales avanzados resultantes de los } \\
\text { productos presentados por los otros grupos. } \\
\text { Selección final de los materiales o familias } \\
\text { de materiales que se caracterizarán. }\end{array}$ & 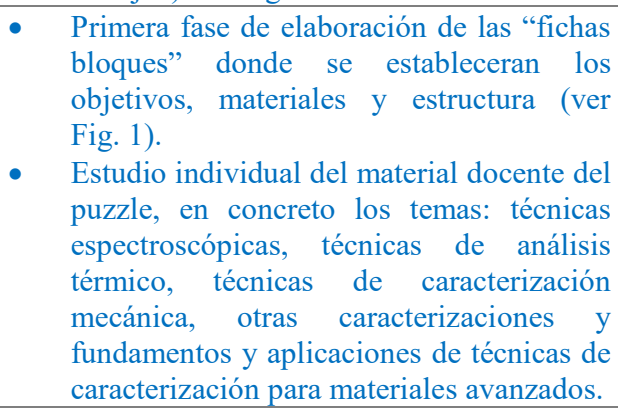 \\
\hline 7 y 8 & $\begin{array}{l}\text { - Clases expositivas para la resolución de } \\
\text { dudas concretas. } \\
\text { - Actividades de autoevaluación. } \\
\text { - Repaso entre todos de las "fichas bloques" } \\
\text { finales propuestas. }\end{array}$ & $\begin{array}{l}\text { - Continuación del estudio individual del } \\
\text { material docente del puzzle, en concreto los } \\
\text { temas: técnicas espectroscópicas, técnicas } \\
\text { de análisis térmico, técnicas de } \\
\text { caracterización mecánica, otras } \\
\text { caracterizaciones y fundamentos y } \\
\text { aplicaciones de técnicas de caracterización } \\
\text { para materiales avanzados. } \\
\text { Reunión del grupo base para explicarse los } \\
\text { materiales del puzzle. } \\
\text { Cierre de la elaboración de las "fichas } \\
\text { bloques" estableciendo las técnicas } \\
\text { principales y secundarias de } \\
\text { caracterización (ver Fig. 1). Entregable 2. }\end{array}$ \\
\hline
\end{tabular}




\begin{tabular}{|c|c|c|}
\hline 9 & $\begin{array}{l}\text { - Procesamiento de los nuevos materiales o } \\
\text { familias de materiales avanzados. }\end{array}$ & - Preparación prueba de evaluación final. \\
\hline 10 a 14 & $\begin{array}{l}\text { - Caracterización de los nuevos materiales o } \\
\text { familias de materiales avanzados. }\end{array}$ & $\begin{array}{l}\text { - Preparación prueba de evaluación final. } \\
\text { - Análisis de resultados. Preparación } \\
\text { memoria del proyecto. Entregable final. } \\
\text { - } \quad \text { Preparación presentación del proyecto. }\end{array}$ \\
\hline 15 & $\begin{array}{l}\text { Entregable final (memoria del proyecto). } \\
\text { - Presentación - defensa pública del } \\
\text { proyecto. } \\
\text { Evaluación final mediante la herramienta } \\
\text { Kahoot. }\end{array}$ & \\
\hline
\end{tabular}

\section{Resultados}

Los resultados conseguidos en el presente curso tras la aplicación de la metodología de Aprendizaje Basado en Proyectos han sido muy buenos. No solo el $100 \%$ de los alumnos que han seguido la asignatura han conseguido superarla, sino que además se ha logrado un mayor grado de adquisición de conocimientos, lo que se traduce en unas notas medias generalmente superiores a las de los anteriores cursos.

Pero con el fin de recabar la opinión de los alumnos y su actitud con respecto a la metodología aplicada, se les pasó una encuesta a la que respondieron todos los alumnos (un total de 12), la cual contenía las siguientes preguntas:

- Pregunta 1: ¿Qué tipo de metodología docente preferirías que se utilizará en las clases de "Estructura y técnicas de caracterización de materiales avanzados"?

- Pregunta 2: ¿Has tenido anteriormente otras asignaturas en las que se utilizará el aprendizaje basado en proyectos?

- Pregunta 3: ¿Piensas que sería interesante que varias asignaturas del Máster se coordinarán en un proyecto común como eje central del aprendizaje?

- Pregunta 4: ¿En la asignatura "Estructura y técnicas de caracterización de materiales avanzados" los objetivos del proyecto se te mostraron claros y concisos desde el primer momento?

- Pregunta 5: ¿En la asignatura "Estructura y técnicas de caracterización de materiales avanzados" la planificación, duración y gestión del proyecto planteada ha sido adecuada?

- Pregunta 6: ¿Piensas que la realización de un proyecto en esta asignatura te ha sido de utilidad para alcanzar conocimientos sobre la materia que consideras que no habrías logrado mediante la docencia tradicional?

- Pregunta 7: ¿Recomendarías este tipo de docencia a otros alumnos?

- Pregunta 8: ¿Consideras que has aprendido más con este nuevo tipo de metodología que en las clases en las que se emplea un método tradicional?

- Pregunta 9: ¿Piensas que te has implicado más en esta asignatura por el tipo de metodología utilizado que en otras con una metodología tradicional?

- Pregunta 10: ¿Estas satisfecho/satisfecha con el proyecto realizado? 
¿Estudias o trabajas? - El aprendizaje basado en proyectos como una experiencia enriquecedora de trabajar y estudiar.

A continuación se muestran gráficamente los resultados de dicha encuesta (ver Fig. 2):
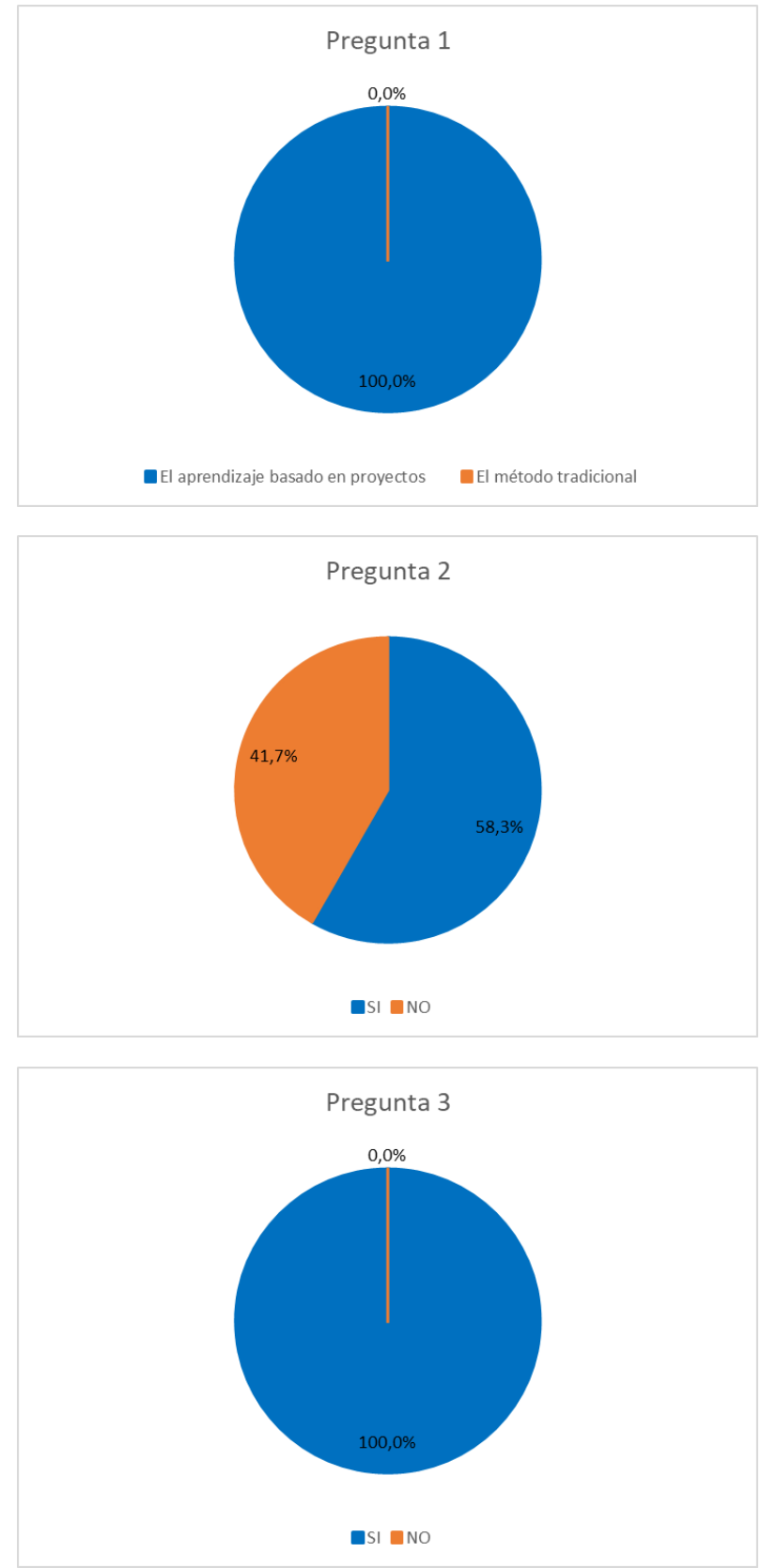

(cc) EY-NC-ND 2019, Universitat Politècnica de València 

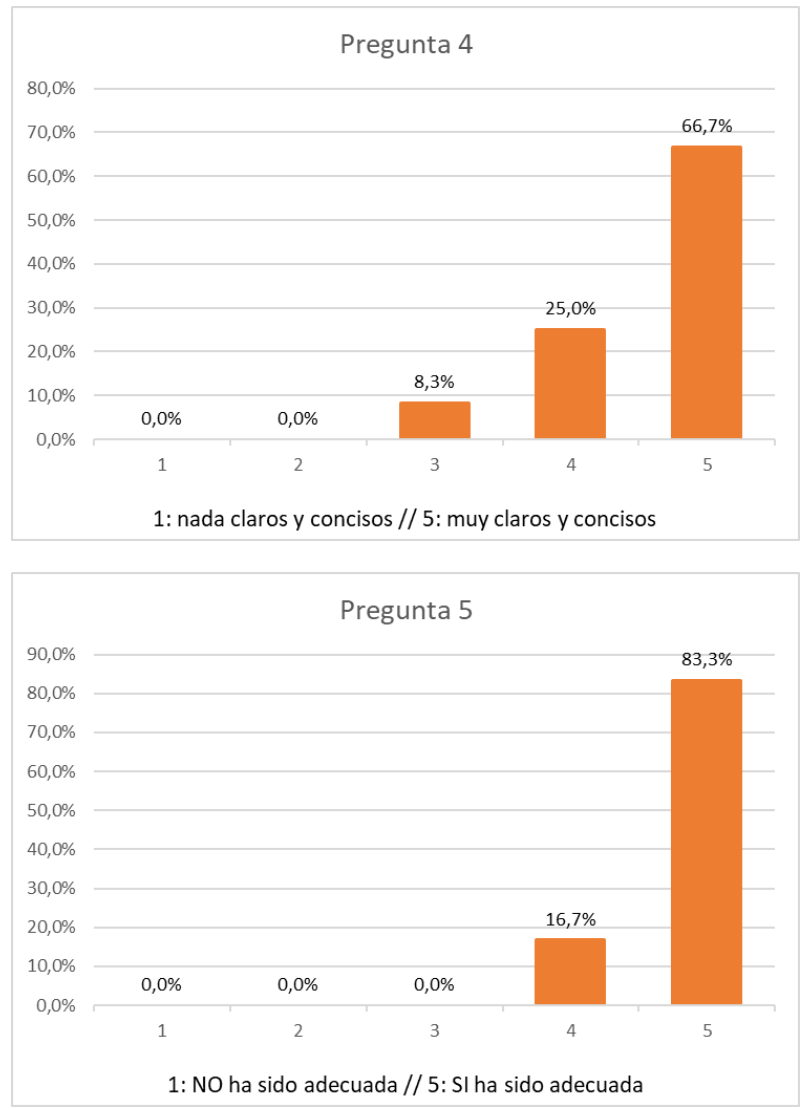

Pregunta 6

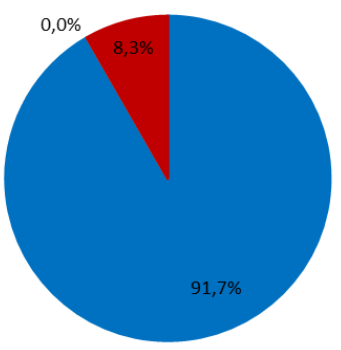

- $\quad$ NI No estoy seguro/a 
¿Estudias o trabajas? - El aprendizaje basado en proyectos como una experiencia enriquecedora de trabajar y estudiar.
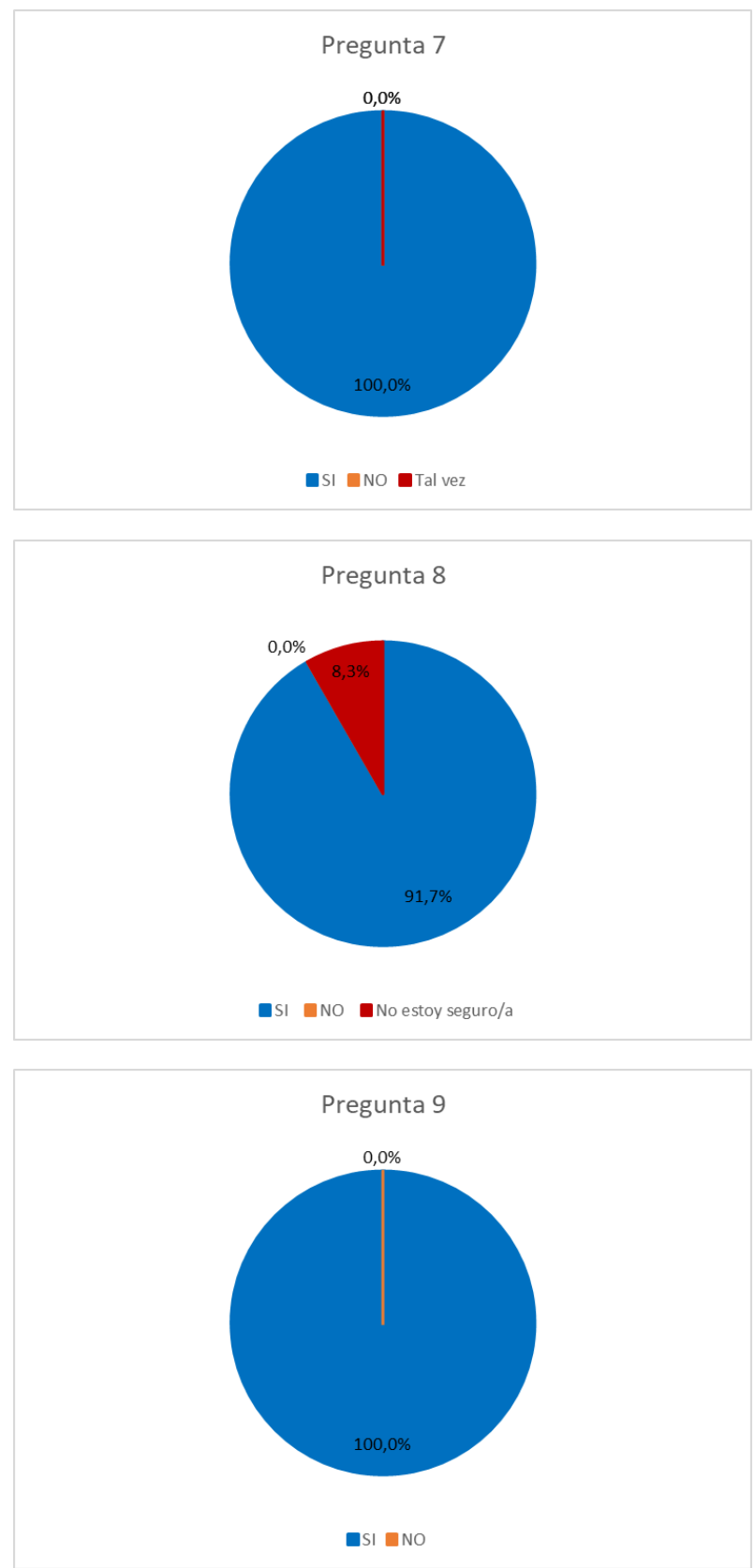

(cc) EY-NC-ND 2019, Universitat Politècnica de València 


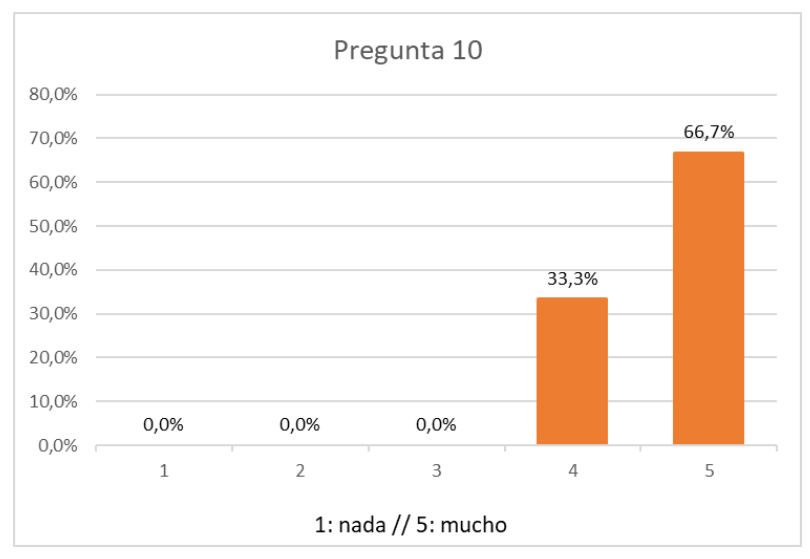

Fig. 2 Resultados de las encuestas de opinión de los alumnos respecto a la metodología PBL.

Para concluir cabe comentar que, analizando las respuestas de los alumnos, se extraen una serie de conclusiones muy enriquecedoras para la actividad docente de los profesores implicados en este cambio metodológico, ya que:

- En general los alumnos están muy satisfechos con la metodología PBL aplicada.

- Los objetivos, planificación, duración y gestión del proyecto les han parecido adecuados.

- La mayoría de los alumnos piensan que han aprendido más y mejor que con un método docente tradicional.

- Todos los estudiantes piensan que su implicación en la asignatura ha sido mucho mayor con la aplicación de la metodología PBL.

- Y por último, todos los alumnos manifiestan que sería interesante que varias asignaturas del Máster se coordinarán en un proyecto común, siendo ésta la intención de los profesores implicados en este proyecto.

\section{Conclusiones}

Como se ha comentado anteriormente, los resultados académicos de los alumnos pueden considerarse muy buenos tras la aplicación de la metodología de Aprendizaje Basado en Proyectos en la asignatura de "Estructura y técnicas de caracterización de materiales avanzados". El 100\% de los alumnos que han seguido la asignatura han conseguido superarla y además se han registrado unas notas medias generalmente superiores a las de los anteriores cursos.

Por otra parte, las encuestas de opinión pasadas a los alumnos denotan no solo que les gusta esta herramienta metodológica, sino lo que es más importante, su sensación de que han aprendido más, mejor y con un mayor grado de implicación.

Como conclusión final, si bien pensamos que el PBL no puede ser una receta mágica que sirva para todo, y que es posible que en determinadas asignaturas no tenga una buena aplicabilidad, para otras muchas sí, y de hecho la idea de combinar varias asignaturas del 
¿Estudias o trabajas? - El aprendizaje basado en proyectos como una experiencia enriquecedora de trabajar y estudiar.

Máster en un proyecto común presuponemos que no sólo es factible, sino que además reportará muchos beneficios desde el punto de vista formativo para nuestros alumnos. Y de hecho postulamos que el PBL debería implantarse también progresivamente en los grados, de forma que, sobre todo en los últimos cursos, tuviera un mayor peso en la actividad de los estudiantes, ya que este tipo de metodología, a parte de las muchas ventajas que promueve, enumeradas en la introducción, realmente creemos que prepara mejor a los alumnos para su inserción en el mundo laboral.

\section{Agradecimientos}

Los autores quieren mostrar su agradecimiento a la UPV y al Instituto de Ciencias de la Educación (ICE) de la UPV por su ayuda y colaboración en la formación del Equipo de Innovación y Calidad Educativa (EICE) denominado GIPA. Así mismo los autores quieren agedecer al Vicerrectorado de Estudios, Calidad y Acreditación de la UPV la concesión del Proyecto de Innovacion y Mejora Educativa (PIME) referencia B18/18, dentro del cual se enmarca el presente trabajo.

\section{Referencias}

BOUD, D. (1985). "Problem-based learning in perspective" en Problem-based Learning in Education for the Professions, vol. 13.

CASCALES MARTÍNEZ, A., CARRILLO GARCÍA, M. y REDONDO ROCAMORA, A. M. (2017). "ABP y tecnología en Educación Infantil" en Pixel-Bit. Revista de Medios y Educación, 50, 201-210.

DE GRAAFF, E. y RAVESTEIJN, W. (2001). "Training complete engineers: global enterprise and engineering education" en European Journal of Engineering Education, 26(4), p. 419-427.

KOLMOS, A., KURU, S., HANSEN, H., ESKIL, T., PODESTA, L., FINK, F. Y SOYLO, A. (2007). Problem based learning: TREE-Teaching and Researc in Engineering in Europe. en Report by Special Interest Group B5,'Problem Based Learning and Project Oriented Learning.

REVERTE BERNABEU, J., GALLEGO, A. J., MOLINA-CARMONA, R. Y SATORRE CUERDA, R. (2007). "El aprendizaje basado en proyectos como modelo docente. Experiencia interdisciplinar y herramientas groupware" en XIII Jornadas de Enseñanza Universitaria de la Informática, JENUI'07, Teruel, Julio 2007. Libro de actas, Madrid: Thomson Paraninfo, 2007, ISBN 978-84-9732-620-9.

SAVIN-BADEN, M. (2000). Problem-Based Learning In Higher Education: Untold Stories. Reino Unido: McGraw-Hill Education.

SCHÖN, D. A. (1987). Educating the reflective practitioner: toward a new design for teaching and learning in the professions. San Francisco (CA, US): Jossey-Bass.

SCHÖN, D. A. (2017). "Educating the reflective practioner: how professionals think in practice en The Journal of Continuing Higher Education, 34(3), p. 29-30. 